\title{
Downregulation of STAT3 and activation of MAPK are involved in the induction of apoptosis by HNK in glioblastoma cell line U87
}

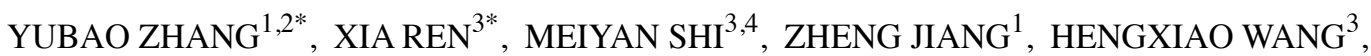 \\ QINGHONG SU ${ }^{3}$, QINGLIN LIU ${ }^{1}$, GANG LI ${ }^{1}$ and GUOSHENG JIANG ${ }^{3}$ \\ ${ }^{1}$ Department of Neurosurgery, Qilu Hospital, Shandong University; ${ }^{2}$ Department of Neurosurgery, \\ Shandong Provincial Hospital, Shandong University; ${ }^{3}$ Key Laboratory for Rare and Uncommon Diseases, Key \\ Laboratory for Tumor Immunology and Chinese Medicine Immunology of Shandong Province, Institute of Basic \\ Medicine, Shandong Academy of Medical Sciences; ${ }^{4}$ University of Jinan, Jinan, Shandong, P.R. China
}

Received April 1, 2014; Accepted June 10, 2014

DOI: $10.3892 /$ or.2014.3434

\begin{abstract}
Honokiol [3,5-di-(2-propenyl)-1,1-biphenyl-2,2-diol; $\mathrm{HNK}$, a natural bioactive molecular compound isolated from the Magnolia officinalis, exhibits potent antitumor activity against a variety of human cancer cell lines. However, few studies have reported the antineoplastic effects of HNK on glioblastoma cells. It remains unknown how apoptosis is induced by HNK in glioblastoma cells and through which associated pathway this compound acts. The present study confirmed that HNK inhibited proliferation of glioblastoma cells by inducing a slight $\mathrm{G}_{0} / \mathrm{G}_{1}$ phase cell cycle arrest and apoptosis. We demonstrated for the first time that HNK triggered apoptosis of glioblastoma cells through both caspase-independent and caspase-dependent pathways, the latter including the extrinsic pathway and intrinsic pathway. Moreover, the inhibition of STAT3 signaling, ERK1/2 as well as activation of the p38 MAPK signaling pathway may be involved in apoptosis induced by HNK in U87 cells. Our findings suggest that HNK treatment could be a promising therapeutic strategy in human glioblastoma.
\end{abstract}

\section{Introduction}

Glioblastoma is the most common and lethal type of primary brain tumor, accounting for $82 \%$ of all malignant glioma

Correspondence to: Dr Gang Li, Department of Neurosurgery, Qilu Hospital of Shandong University, 107 Wen Hua Xi Road, Jinan, Shandong 250012, P.R. China

E-mail: ligang2008121@sohu.com

Dr Guosheng Jiang, Department of Hemato-Oncology, Institute of Basic Medicine, Shandong Academy of Medical Sciences, 18877 Jingshi Road, Jinan, Shandong 250062, P.R. China

E-mail: jianggsh@hotmail.com

*Contributed equally

Key words: apoptosis, glioblastoma, STAT3, MAPK cases (1). Despite multimodal treatment including surgical resection, adjuvant TMZ-based chemotherapy and radio therapy, the prognosis of glioblastoma remains poor, with a median overall survival time of only 15 months from the time of diagnosis (2). It is now commonly recognized that the rapid proliferation of glioblastoma cells, coupled with its widely invasive growth and persistently aggressive biological behavior, lead to treatment failure and tumor recurrence (3). Therefore, the development of novel agents with promising therapeutic results and minimized toxicities for the treatment of glioblastoma is urgently needed.

Honokiol [(3,5-di-(2-propenyl)-1,1-biphenyl-2,2-diol; $\mathrm{HNK}]$ is a natural bioactive molecular compound isolated from the Magnolia officinalis, a well-known Chinese traditional medicine that has been used for thousands of years in the treatment of anxiety, thrombotic stroke and gastrointestinal complaints. Accumulating studies have demonstrated that HNK exerts multiple pharmacological activities including anti-inflammatory, anti-bacterial, anti-angiogenic, anti-thrombocytic and anti-oxidative activity (4). Moreover, extensive mechanistic and preclinical studies have indicated that HNK exhibits potent antitumor activity against a variety of human cancer cell lines (5-8), without appreciable toxicity (9). The antitumor properties of $\mathrm{HNK}$ in relation to cell cycle arrest $(7,8)$, apoptosis $(5,6)$ and paraptosis $(10)$, or autophagy (11) have been well characterized. However, few studies have reported the antineoplastic effects of HNK on glioblastoma cells and it is not known how apoptosis is induced by HNK on glioblastoma cells and through which associated pathway this compound acts. Wang et al (12) reported that HNK could effectively cross blood-brain barrier (BBB) and blood-cerebrospinal fluid barrier (BCSFB) and inhibit brain tumor growth in rat $9 \mathrm{~L}$ intracerebral gliosarcoma model and human U251 xenograft glioma model. Another study verified that HNK exerts an anticancer effect on T98G human glioblastoma cells through the induction of apoptosis and inhibition of adhesion molecules ICAM-1 and VCAM-1 expression (13).

In the present study, we investigated the therapeutic potential of HNK against U87 cells in vitro. This study demonstrated 
that HNK inhibits growth of glioblastoma cells by causing a slight $G_{0} / G_{1}$ phase cell cycle arrest and inducing apoptosis via both caspase-dependent and -independent pathways. We also demonstrated that HNK exerts antiproliferative activity on glioblastoma cells through the inhibition of signal transducers and activator of transcription 3 (STAT3) signaling, and ERK1/2 signaling pathway, as well as through activation of the p38 MAPK signaling pathway. Our findings suggest that HNK treatment could be a promising therapeutic strategy in human glioblastoma.

\section{Materials and methods}

Cell lines and reagents. The human glioma cell lines U87, U251 and T98G were grown in Dulbecco's modified Eagle's medium (DMEM) supplemented with $10 \%$ fetal calf serum (FCS), $100 \mathrm{IU} / \mathrm{ml}$ penicillin and $100 \mathrm{IU} / \mathrm{ml}$ streptomycin in a $5 \% \mathrm{CO}_{2}$ atmosphere at $37^{\circ} \mathrm{C}$. HNK with a purity of up to $99.5 \%$ was kindly provided by Professor Xiao Wang (Shandong Analysis and Test Center, Shandong Academy of Sciences), and stock solutions were prepared in dimethyl sulfoxide (DMSO) at a concentration of $100 \mathrm{mg} / \mathrm{ml}$ and dissolved in DMEM medium prior to use. Cell Counting Kit-8 (CCK8) was purchased from Dojindo Laboratories (Kumamoto, Japan). Propidium iodide (PI), Rhodamine 123 (Rh123) were purchased from Sigma. Hoechst 33342, p38 inhibitor (SB203580), JNK1/2 inhibitor (SP600125), MEK-1/2 inhibitor (U0126) and caspase inhibitor (Z-VAD-fmk) were obtained from Beyotime Biotechnology Inc. (Nantong, China). Annexin V (FITC) apoptosis detection kit was obtained from BD Biosciences (San Diego, CA, USA). Antibodies against Bax, Bcl-2, cleaved caspase-3,-9 and -8, Fas, FasL, survivin, phospho-ERK1/2, ERK1/2, phospho-p38, p38, phospho-JNK were purchased from Cell Signaling Technology (Beverly, MA, USA). JNK antibody was purchased from Santa Cruz. Horseradish peroxidase-labeled IgG anti-mouse and anti-rabbit antibodies were supplied by Proteintech Group Inc. (Chicago, IL, USA). GAPDH-HRP antibody and anti-LC3 $\alpha \beta$ antibody were obtained from Epitomics.

Cell proliferation assay. Cell proliferation was analyzed by Cell Counting Kit-8 (CCK-8; Dojindo Laboratories), according to the manufacturer's protocol, as previously described (14). Cell viability was measured using trypan blue exclusion and live cells were enumerated. Cell counts were expressed as mean \pm standard deviation (SD).

Cell cycle distribution. After exposure to $10 \mu \mathrm{g} / \mathrm{ml} \mathrm{HNK}$ for different exposure durations, cells $\left(3 \times 10^{5}\right)$ were washed twice with ice-cold phosphate-buffered saline (PBS), and fixed in cold $75 \%$ ethanol at $4^{\circ} \mathrm{C}$ for at least $24 \mathrm{~h}$. Then, the cells were rinsed with PBS, resuspended in $1 \mathrm{ml}$ of cell cycle buffer $(0.38 \mathrm{~mm}$ Na-citrate, $0.5 \mathrm{mg} / \mathrm{ml} \mathrm{RNase} \mathrm{A,} \mathrm{and} 20 \mu \mathrm{g} / \mathrm{ml}$ propidium iodide) at room temperature for $40 \mathrm{~min}$ and the cells were analyzed using an EPICS XL flow cytometer with EXPO32 ${ }^{\mathrm{TM}}$ ADC software (Beckman Coulter, Miami, FL, USA).

Reverse transcription-PCR assays. Cells were incubated with $10 \mu \mathrm{g} / \mathrm{ml} \mathrm{HNK}$ for 24 and $48 \mathrm{~h}$. Then, total RNA was extracted using TRIzol (Invitrogen, Carlsbad, CA, USA) according to the manufacturer's instructions. RNA purity determination, cDNA synthesis, and reverse transcription-PCR (RT-PCR) were performed as previously described (16). The primers used were all synthesized by Sangon Co., Ltd. (Shanghai, China) and the sequences were as follows: cyclin E (301 bp) sense, 5'-ATACAGACCCACAGAGACAG-3' and antisense, 5'-TGCCATCCACAGAAATACTT-3'; p27 (270 bp) sense, 5'-ATGTCAAACGTGCGAGTGTC-3' and antisense, 5'-TCT GTAGTAGTCGGGCAA-3'; P21 (341 bp) sense, 5'-CCCTCC TGGCTCTTGATACCC-3' and antisense, 5'-TGCCCTTCTT CTTGTGTGTCCC-3'.

Detection of apoptosis. After HNK treatment for 12 or $24 \mathrm{~h}$, cells were observed under inverted microscope and photographed. Then, the cells were collected and washed with PBS, fixed with $4 \%$ paraformaldehyde for $15 \mathrm{~min}$, and stained with Hoechst $33342(10 \mu \mathrm{g} / \mathrm{ml})$ for $5 \mathrm{~min}$ at room temperature in the dark. After washing three times, cells were resuspended by PBS. Stained nuclei were observed by a fluorescence microscope and photographed.

HNK-induced U87 cells undergoing apoptosis were further determined using an Annexin V/FITC apoptosis kit. Briefly, after exposure to $10,20 \mu \mathrm{g} / \mathrm{ml}$ of $\mathrm{HNK}$ for different time points, the cells were harvested, washed twice with cold PBS and resuspended in $100 \mu \mathrm{l} 1 \mathrm{X}$ binding buffer containing $5 \mu \mathrm{l}$ of Annexin V incubation reagent and $10 \mu \mathrm{l}$ of PI. After incubation at room temperature in the dark for $15 \mathrm{~min}$, analysis was carried out using a Beckman Coulter EPICS XL cytometer.

Caspase inhibition. To evaluate the involvement of caspases in HNK-induced apoptosis, cells were preincubated with the pan-caspase inhibitor Z-VAD-fmk $(25 \mu \mathrm{M})$ for $1 \mathrm{~h}$ prior to incubation with HNK. Annexin V/FITC analysis was performed as previously described.

Analysis of mitochondrial membrane potential $(\Delta \Psi m) . \Delta \Psi \mathrm{m}$ was assessed using fluorescent dye Rh123 and flow cytometric analysis. Briefly, after HNK treatment for $6 \mathrm{~h}$, non-adherent cells were collected, and attached cells were trypsinized and washed twice with PBS. Cells $\left(1 \times 10^{6}\right)$ in different groups were incubated with $10 \mathrm{mg} / \mathrm{ml} \mathrm{Rh} 123$ for $30 \mathrm{~min}$ at $37^{\circ} \mathrm{C}$. Then, cells were washed twice and resuspended in PBS followed by flow cytometric analysis. The change in the mean fluorescence intensity reflects the modification of $\Delta \Psi \mathrm{m}$, which drives the uptake and accumulation of Rh123 in the mitochondria.

Western blot analysis. Cells were lysed with lysis buffer (50 mM Tris- $\mathrm{HCl} \mathrm{pH}$ 7.4, $150 \mathrm{mM} \mathrm{NaCl}, 1 \mathrm{mM}$ PMSF, $1 \mathrm{mM}$ EDTA, $1 \%$ Triton X-100, 0.5\% deoxycholate, $1 \mu \mathrm{g} / \mathrm{ml}$ leupeptin, $2 \mu \mathrm{g} / \mathrm{ml}$ aprotinin, $1 \mathrm{mM} \mathrm{Na} \mathrm{VO}_{4}$ and $0.1 \% \mathrm{SDS}$ ). Protein concentration of each sample was determined by the Bradford protein assay. Equal quantities of protein were subjected to SDS-PAGE and were then transferred to PVDF membranes. The membranes were blocked with $5 \%$ milk for $1 \mathrm{~h}$ and incubated with primary rabbit monoclonal antibodies against cleaved caspase-3 (1:1,000), -8 and -9, Fas, FasL, Bcl-2 (1:1,000) and Bax (1:1,000), survivin, phospho-ERK1/2, ERK1/2, phospho-p38, p38, phospho-JNK, JNK, phospho-STAT3 and STAT3 overnight at $4{ }^{\circ} \mathrm{C}$ followed by incubation with HRP-conjugated secondary antibodies for $1 \mathrm{~h}$. 

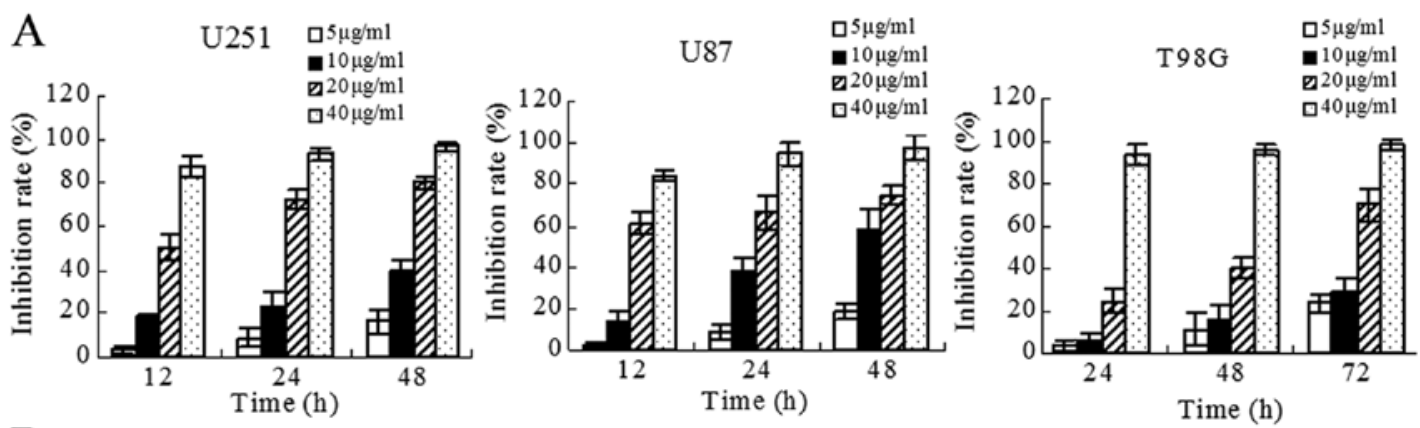

B
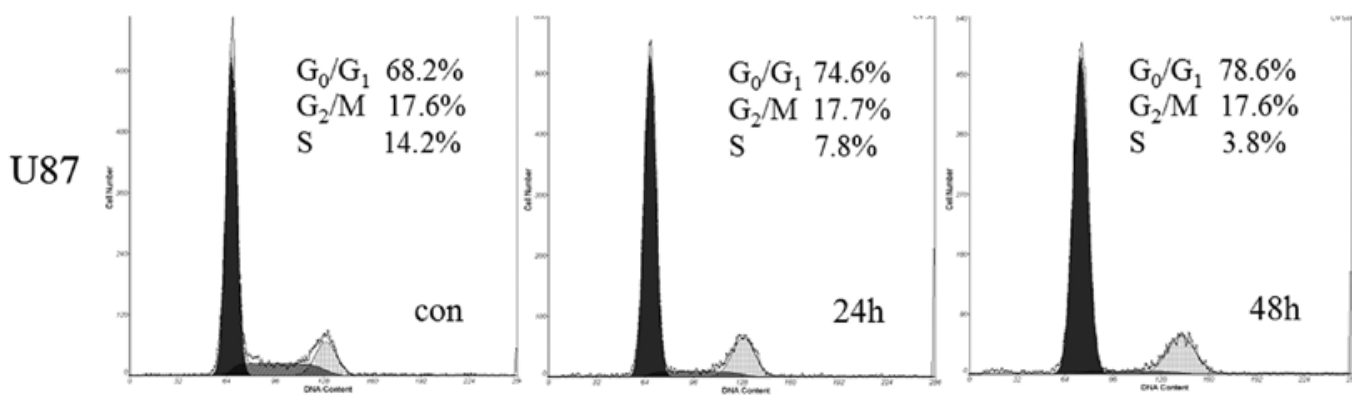

$\mathrm{C}$
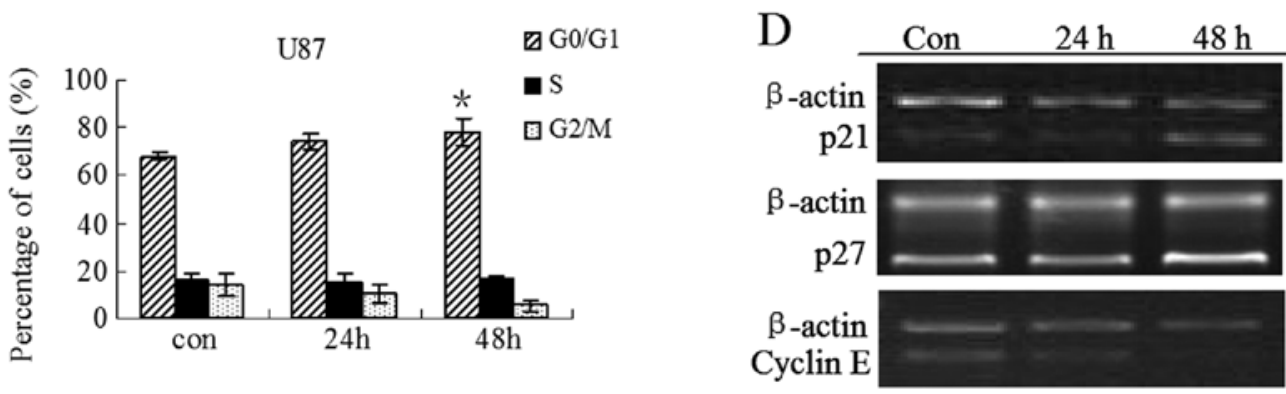

Figure 1. Evaluation of cell growth and cell cycle distribution of glioblastoma cells after treatment with HNK. (A) U251, U87 and T98G cells were incubated with $\mathrm{HNK}$ at a concentration of $5,10,20,40 \mu \mathrm{g} / \mathrm{ml}$ for the indicated time points and the number of surviving cells was determined using CCK8 assay as described in Materials and methods. (B) The cells were treated with $10 \mu \mathrm{g} / \mathrm{ml} \mathrm{HNK}$ for 0, 24 and $48 \mathrm{~h}$, they were then centrifuged, incubated in $70 \%$ ethanol, stained with PI, and then the DNA content was analyzed by flow cytometry as described in Materials and methods. One representative experiment of three is shown. (C) The histograms show that there was a slight increase of $G_{0} / G_{1}$ population with concomitant decrease of $S$ and $G_{2} / M$ population after treatment with HNK. All results are described as means \pm SD of three independent experiments. ${ }^{*} \mathrm{P}<0.05$ compared with control. (D) U87 cells were treated with HNK for 24 and $48 \mathrm{~h}$, then the mRNA expression of p21, p27 and cyclin E was detected by RT-PCR. Each amplification was performed at least three times. Semiquantitative analysis was performed using the Image $J$ software program.

The protein bands were visualized using Immobilon Western Chemiluminescent HRP substrate (Millipore, Billerica, MA, USA) and pictured by LAS-4000 mini luminescent image analyzer (Fujifilm, Tokyo, Japan).

Statistical analysis. All experiments were performed in triplicate. Data are represented as means \pm standard deviation (SD). Statistical analyses were performed using Student's t-test or one-way ANOVA. In all analyses, $\mathrm{p}<0.05$ was considered to indicate a statistically significant difference.

\section{Results}

HNK inhibits growth in glioblastoma cells. To determine the effect of HNK on the cellular proliferation in glioblastomas, we performed dose-response and time-course studies in three established cell lines, U251, U87 and T98G. DMSO, which was used to dissolve HNK, served as the control. As shown in Fig. 1A, HNK markedly inhibited growth of all three cell lines in a dose- and time-dependent manner from 5 to $40 \mu \mathrm{g} / \mathrm{ml}$
HNK at 12-72 h. Based on the results obtained in terms of cell growth inhibition, we found that U87 cells were most sensitive to HNK and were selected for the following steps of our study. Treatment of U87 cells with $40 \mu \mathrm{g} / \mathrm{ml} \mathrm{HNK}$ for $12 \mathrm{~h}$ resulted in a great loss of viability (as shown by trypan blue staining), suggesting that treatment with HNK caused cell necrosis at this concentration. In comparison, the viability of cells treated with $20 \mu \mathrm{g} / \mathrm{ml}$ remained at $92 \%$ (data not shown). Therefore, the cells treated with 10 and $20 \mu \mathrm{g} / \mathrm{ml} \mathrm{HNK}$ were adopted to perform the subsequent experiments.

HNK induces a slight $G_{0} / G_{1}$ arrest in U87 cells. To determine whether the growth inhibitory effect of HNK is due to the arrest of cell cycle, the distribution of cellular DNA content in U87 cells was examined in the presence of $\mathrm{HNK}$ at $10 \mu \mathrm{g} / \mathrm{ml}$ for 24 and $48 \mathrm{~h}$. Representative flow histograms and quantitative data depicting cell cycle distribution in U87 cultures are shown in Fig. 1B and C. HNK caused a slight accumulation in $\mathrm{G}_{0} / \mathrm{G}_{1}$ phase cell population ( 68.2 in control vs. 74.6 and $78.6 \%$ in cells treated for 24 and $48 \mathrm{~h}$, respectively), accompanied 

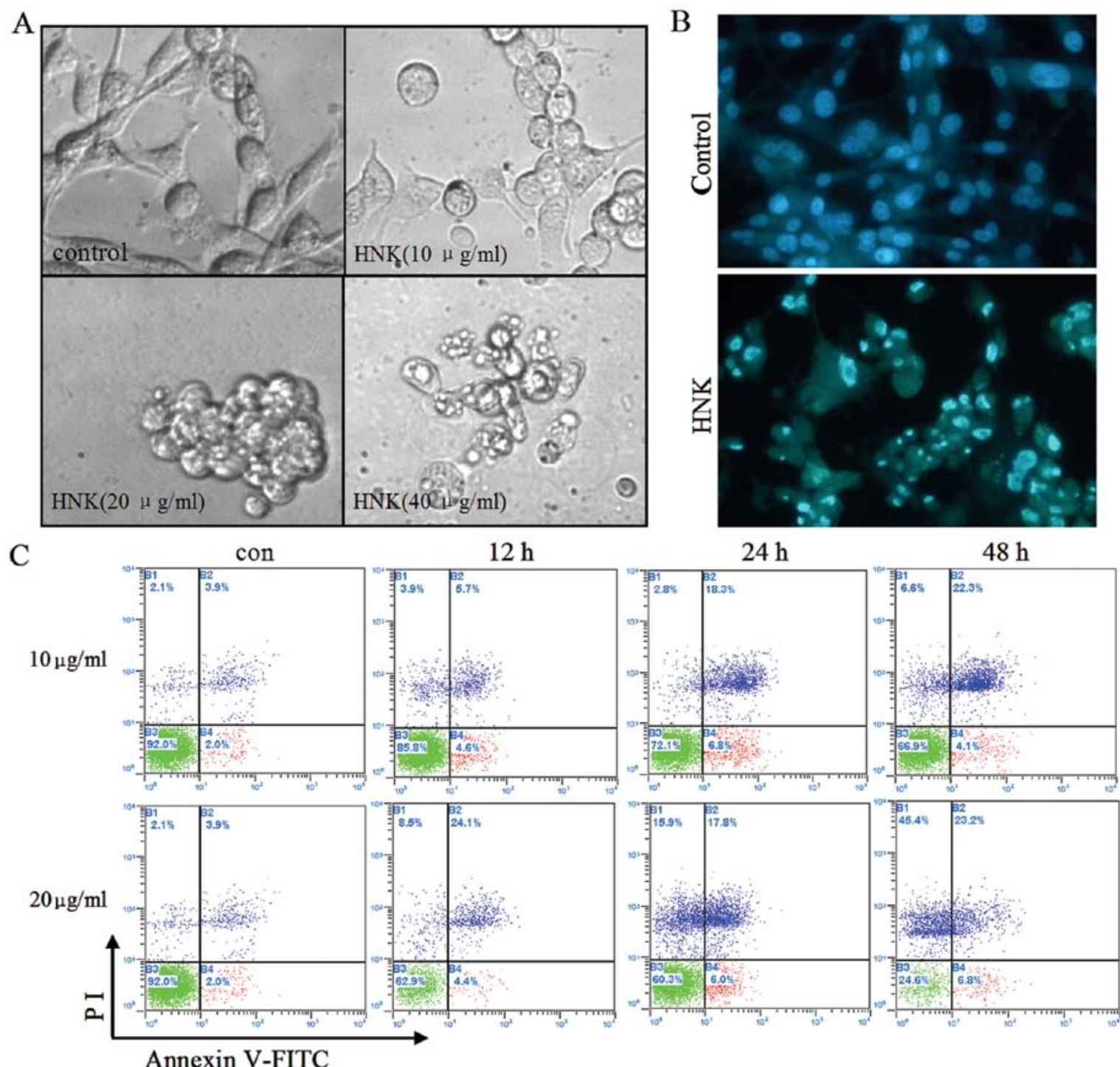

Annexin V-FITC

Figure 2. HNK induces apoptosis in U87 cells. (A) HNK-induced morphological changes in U87 cells. U87 cells were treated with 10, 20, 40 $\mu \mathrm{g} / \mathrm{ml} \mathrm{HNK}$ for $12 \mathrm{~h}$, and were then observed under inverted microscope (magnification, $\mathrm{x} 400$ ). (B) Cells exposed to $10 \mu \mathrm{g} / \mathrm{ml} \mathrm{HNK}$ for $24 \mathrm{~h}$ were fixed and stained with Hoechst 33342. Condensed or fragmented nuclei were considered as apoptotic cells (magnification, x200). (C) HL-60 cells were treated with 10 or $20 \mu \mathrm{g} / \mathrm{ml}$ HNK for the indicated times, stained with Annexin V and PI, and then analyzed by flow cytometry. Cells in the lower right hand quadrant (Annexin $\left.\mathrm{V}^{+} / \mathrm{PI}\right)$ correspond to early apoptotic cells, whereas cells appearing in the right upper quadrant (Annexin $\mathrm{V}^{+} / \mathrm{PI}^{+}$) correspond to late apoptotic cells.

by a concomitant reduction in S-phase cell population $(14.2$ in control vs. 7.8 and $3.8 \%$ in HNK-treated cells for 24 and $48 \mathrm{~h}$, respectively). However, the slight cell cycle block is not completely in parallel to the marked inhibition of cell proliferation at the same time points, indicating that a $\mathrm{G}_{0} / \mathrm{G}_{1}$ phase cell cycle arrest may be partly associated with cell growth inhibition by HNK.

In order to further explore the cell cycle arrest in U87 cells under the influence of HNK, the mRNA expression of cell cycle regulatory genes involved in $\mathrm{G}_{1} / \mathrm{S}$ transition and $\mathrm{S}$ phase was detected by RT-PCR (Fig. 1D). The results indicated that cyclin E was significantly reduced after HNK treatment for $48 \mathrm{~h}$. In contrast, the expression of p21 and p27 was pronouncedly upregulated compared to the control at this time point, while there was no detectable change at $24 \mathrm{~h}$. Altered expression of cyclin E, p21 and p27 may account for an arrest in $G_{0} / G_{1}$ phase, in line with the change of cell cycle distribution at the same time points.
HNK induces apoptosis in U87 cells. To further clarify whether the growth inhibitory effect of HNK was associated with apoptosis, in the first step, morphological changes of U87 cells were observed under an inverted light microscope and an inverted fluorescence microscope after Hoechst 33342 staining. It was noted that after $10 \mu \mathrm{g} / \mathrm{ml} \mathrm{HNK}$ treatment for $12 \mathrm{~h}$, the number of U87 cells moderately decreased and a proportion of the cells shrank, detached from the culture plate and became round. Furthermore, cells treated with $20 \mu \mathrm{g} / \mathrm{ml} \mathrm{HNK}$ displayed greater morphological changes. The cells were completely detached from the culture plate and apoptotic bodies were formed. Even at the concentration of $40 \mu \mathrm{g} / \mathrm{ml}$, most of the cells underwent necrosis characterized by structural properties such as a loss of membrane integrity, cellular swelling and organelle degradation (Fig. 2A). Trypan blue staining further confirmed the result (data not shown). Accordingly, Hoechst 33342 staining results showed that the nuclei of control cells were round and large in size, exhibiting 


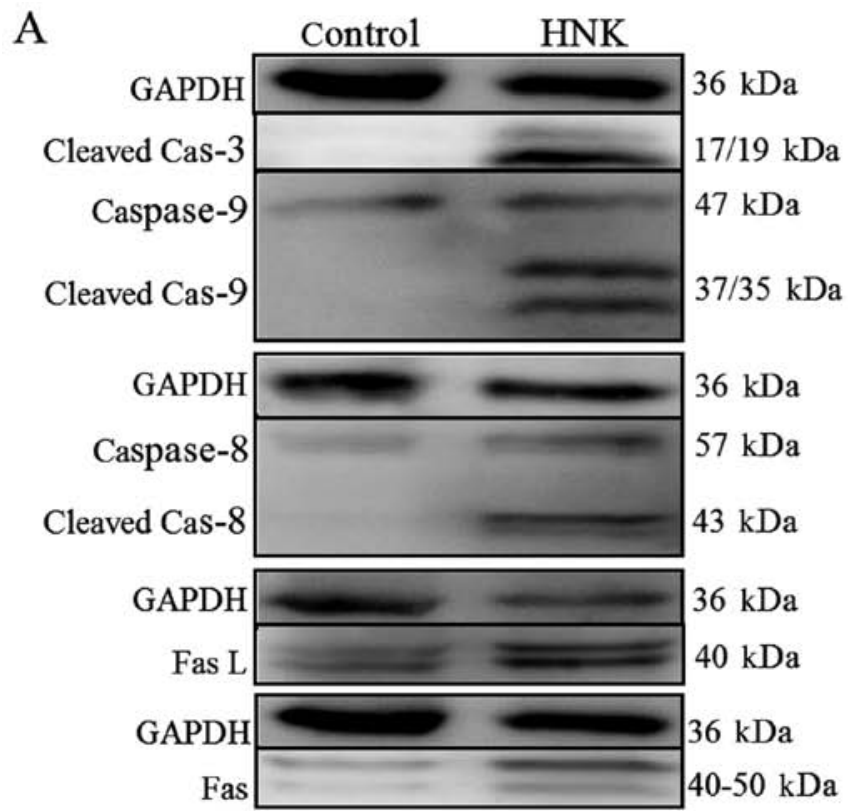

B

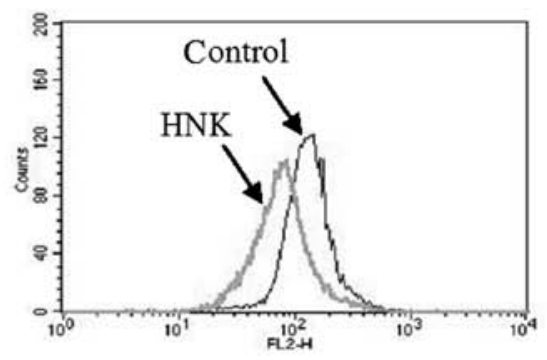

C

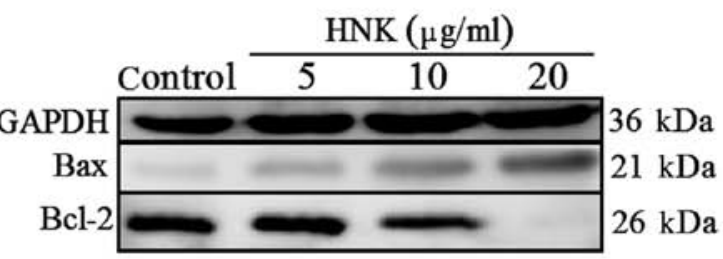

Figure 3. HNK induces caspase-dependent apoptosis. (A) U87 cells were treated with $10 \mu \mathrm{g} / \mathrm{ml} \mathrm{HNK}$ for $24 \mathrm{~h}$, then the cell lysates were subjected to western blotting to determine protein level of caspase-8 and -9, cleaved caspase-3, Fas and FasL. GAPDH was used as the loading control. Three independent studies yielded equivalent results and representative blots are shown. (B) Changes in $\Delta \Psi \mathrm{m}$ in HNK-treated U87 cells as determined by flow cytometry analysis. Fluorescence intensity shifts from a higher to lower level indicate a decrease in $\Delta \Psi \mathrm{m}$. (C) U87 cells were treated with $5,10,20 \mu \mathrm{g} / \mathrm{ml} \mathrm{HNK}$ for $24 \mathrm{~h}$, then the cell lysates were subjected to western blotting to determine protein level of Bax and Bcl-2. GAPDH was used as the loading control. Three independent studies yielded equivalent results and representative blots are shown.

homogeneous blue fluorescence. By contrast, parts of cells treated with $10 \mu \mathrm{g} / \mathrm{ml} \mathrm{HNK}$ for $24 \mathrm{~h}$ exhibited condensed or fragmented nuclei which is characteristic of cell apoptosis (Fig. 2B). In order to confirm apoptosis induced by HNK, U87 cells treated with $10,20 \mu \mathrm{g} / \mathrm{ml} \mathrm{HNK}$ for different durations were analyzed for Annexin V/PI double staining by flow cytometry. A time-dependent study indicated that HNK at $10 \mu \mathrm{g} / \mathrm{ml}$ induced $10.3,25.1$ and $26.4 \%$ of total apoptotic U87 cells following 12,24 and $48 \mathrm{~h}$ treatment. While at $20 \mu \mathrm{g} / \mathrm{mg}$, apoptotic cells reached 28.5, 23.3 and $30 \%$ after 12, 24 and $48 \mathrm{~h}$ treatment, accompanied by elevated necrotic cells for 8.5 , 15.3 and $45.4 \%$ (Fig. 2C).

$H N K$ results in the activation of caspase-3, -8 and -9. Caspase family of cysteinyl-proteases plays the key role in the initiation and execution of programmed cell death (16). Thus, the activities of effector caspase (caspase-3) and initiator caspase (caspase- 8 and -9) were investigated by western blotting. Fig. 3A shows that the cleaved fragments of caspase- $3,-8$ and -9 , which indicate the activation of caspase- $3,-8$ and -9 , were observed to be more evident after treatment of U87 cells with $10 \mu \mathrm{g} / \mathrm{ml}$ HNK for $24 \mathrm{~h}$ in comparison with those in control cells. The results suggest that both the extrinsic pathway (death receptor pathway) and intrinsic pathway (mitochondrial pathway) were involved in HNK-induced apoptosis.

HNK-induced apoptosis is mediated through intrinsic pathways. To further address the intrinsic pathway, mitochondrial membrane potential $(\Delta \Psi \mathrm{m})$ was monitored by flow cytometry using Rh123. As shown in Fig. 3B, control cells exhibited strong staining for Rh123, whereas treatment with HNK for $6 \mathrm{~h}$ led to the cell population shifting to the left, indicating that HNK induced a marked loss of $\Delta \Psi \mathrm{m}$. Such findings were consistent with the activation of caspase-9, which is often the result of disruption of $\Delta \Psi \mathrm{m}$.

It is well known that the Bcl-2 family of proteins represents key regulators of the mitochondrial apoptotic pathway (17). The influence of HNK on the expression of Bax and Bcl-2 was examined. As shown in Fig. 3C, treatment of U87 cells with $\mathrm{HNK}$ for $24 \mathrm{~h}$ led to an upregulation in the expression of Bax and a concurrent downregulation in the expression of Bcl-2 in a dose-dependent manner, accordingly leading to an increase in the $\mathrm{Bax} / \mathrm{Bcl}-2$ ratio (data not shown). Taken together, the data suggest that the mitochondrial pathway is likely to be involved in the apoptotic process induced by HNK in glioblastoma cells.

HNK-induced apoptosis is mediated through Fas-mediated extrinsic pathways. To characterize the role of the extrinsic pathway in HNK-induced apoptosis, we detected protein expression of Fas and FasL by western blotting. The results indicated that HNK induced a significant elevation in Fas and FasL (Fig. 3A). The data presented herein suggest that activation of the extrinsic Fas-related pathway may also play a role in HNK-induced apoptosis.

The caspase-independent pathway is also involved in HNK-induced apoptosis. A previous study revealed that both caspase-dependent and -independent mechanisms are involved in HNK-induced apoptosis in human multiple myeloma (18). To verify whether caspases are necessary in apoptosis induced by HNK in U87 cells, we pretreated cells with pan-caspase inhibitor Z-VAD-fmk ( $25 \mu \mathrm{M})$ for $1 \mathrm{~h}$ prior to HNK exposure, and checked for signs of apoptosis. As shown in Fig. 4, we 
A

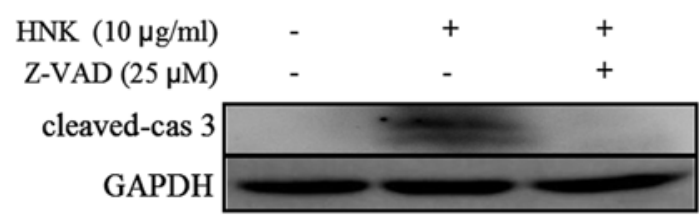

B

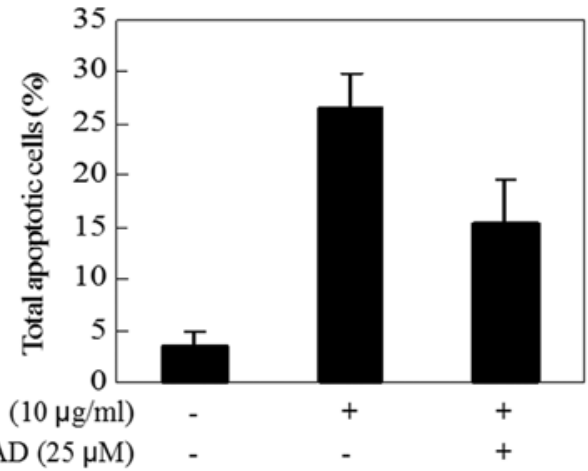

Figure 4. HNK induces caspase-independent apoptosis. U87 cells were pre-incubated with the pan-caspase inhibitor Z-VAD-fmk (25 $\mu \mathrm{M}$ ) for $1 \mathrm{~h}$ prior to HNK exposure, and were then stained with Annexin V and PI and analyzed by flow cytometry.

A

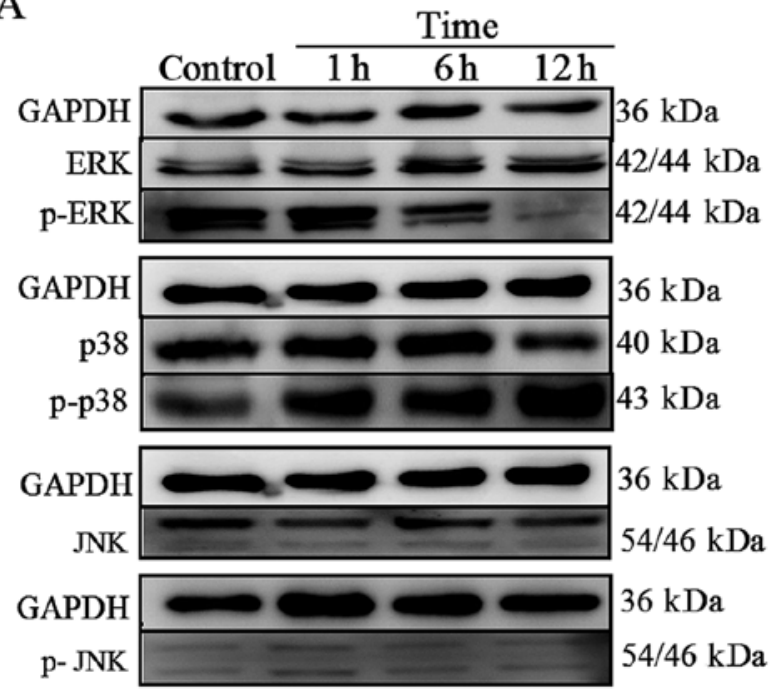

B
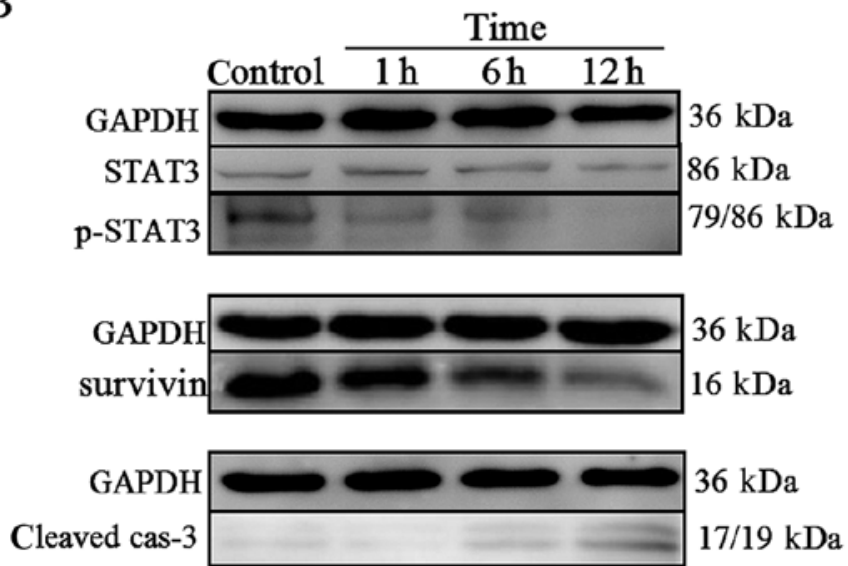

Figure 5. HNK modulates growth and survival signaling pathways in U87 cells. (A) U87 cells were treated with $10 \mu \mathrm{g} / \mathrm{ml} \mathrm{HNK}$ for 1,6 and $12 \mathrm{~h}$, then wholecell lysates were subjected to western blotting to determine phosphorylation and protein expression of MAPK (ERK1/2, p38, JNK). (B) U87 cells were treated with $10 \mu \mathrm{g} / \mathrm{ml} \mathrm{HNK}$ for 1,6 and $12 \mathrm{~h}$, then whole-cell lysates were subjected to western blotting to determine phosphorylation and protein expression of STAT3 and its target gene, survivin. GAPDH was used as the loading control. Three independent studies yielded equivalent results and representative blots are shown.

found that although caspase-3 activity was almost completely blocked by the pan-caspase inhibitor Z-VAD-FMK, the inhibitor did not block HNK-induced apoptosis. These results suggest that caspase-independent pathways may be involved in HNK-induced apoptosis.

The MAPK signaling pathway is involved in HNK-induced cell death in U87 cells. To define the intracellular mechanisms by which HNK affects human glioblastoma cell survival in vitro, we analyzed the activation of intracellular pathways related to glioblastoma development MAPK pathways. We performed western blotting to detect the protein phosphorylation state of ERK, p38 and JNK in control cells and in cells treated for 1, 6 and $12 \mathrm{~h}$ with HNK. There were no detectable changes in expression of total ERK, p38, JNK and phosphorylated-JNK protein level (Fig. 5A). In contrast, phosphorylation of ERK was reduced within $12 \mathrm{~h}$ from the onset of HNK treatment. Also, the treatment caused a significant increase in the activation of p38. These findings strongly indicated that activated p38 and abrogated ERK1/2 may both be involved in the HNK-induced apoptosis of U87 cells (Fig. 5A).

The STAT3 signaling pathway is also involved in HNK-induced apoptosis. STAT3, a signal transducer and activator of transcription 3, is overexpressed in gliomas (19). Its involvement in tumor genesis can be attributed to its ability to induce cell proliferation and inhibit apoptosis (20). Therefore, to investigate whether the STAT3 signaling pathway was involved in HNK-induced U87 cells death, we performed western blotting to assess the protein phosphorylation state and the protein expression of survivin (its target gene). The results indicated that HNK suppressed the phosphorylation of STAT3 on Tyr705 for activation, without affecting the total abundance of STAT3 (Fig. 5B). In parallel, survivin, as one of the downstream targets of STAT3 (21), was downregulated significantly, in line with the upregulation of cleaved caspase-3 expression (Fig. 5B). Hence, STAT3 signaling pathway may contribute to apoptosis induced by HNK. 


\section{Discussion}

HNK has been shown to have a broad spectrum of biological activities, including anti-inflammatory, anti-angiogenic, anti-thrombocytic and anticancer activity, based on recent discoveries in basic and translational research (4). Despite numerous studies being conducted to investigate the effect of HNK on a variety of human cancer cell lines, specific information on the glioblastoma lineage remains scant $(12,13)$. In the present study, we confirmed that HNK exhibits strong dose- and time-dependent anticancer activity in all three glioblastoma cell lines: U87, U251 and T98G cells. According to the inhibition rate curve, we found that the U87 cell line was the most sensitive to HNK. Thus, the U87 cell line was selected to perform the following experiments.

To gain insight into the molecular mechanism involved in the growth arrest of glioblastoma by HNK, we first detected the cell cycle distribution and found that HNK particularly enhanced the amount of $\mathrm{G}_{0} / \mathrm{G}_{1}$ phase cells and diminished the amount of S-phase cells, as demonstrated previously in other cell lines $(8,9)$. In parallel to the alteration of cell cycle distribution, we observed the downregulation of cyclin E mRNA and upregulation of p21 and p27 mRNA. Collectively, these may account for HNK-mediated $\mathrm{G}_{0} / \mathrm{G}_{1}$ phase cell cycle arrest in U87 cells since cyclin $E$ is essential for the $\mathrm{G}_{1}$-to-S phase transition (22), while the Cdk inhibitor p21 and p27 regulates G1-S transition by binding to and inhibiting kinase activity of $\mathrm{Cdk} /$ cyclin complexes (23). It is interesting to note, however, that a slight $\mathrm{G}_{0} / \mathrm{G}_{1}$ arrest does not concur with marked growth inhibition by HNK at the same concentration and time point, indicating that there are at least one other mechanism responsible for the proliferation inhibition of U87 cells.

In the present study, the cell morphological changes and Annexin V/PI staining results, together with activation of caspase-3, confirmed pronounced apoptosis induced by HNK in accordance with earlier studies on the effect of HNK in human glioma cells $(12,13)$, also in parallel to marked growth inhibition by HNK at the same time point. It suggests that the apoptosis may primarily contribute to the antiproliferative activity of HNK on U87 cells.

It is well known that classical apoptosis is mediated by two major pathways, known as the intrinsic (mitochondrial-mediated) and the extrinsic (receptor-mediated) pathway (24). The extrinsic pathway is initiated by the binding of a death ligand [e.g., FasL, tumor necrosis factor (TNF)-related apoptosisinducing ligand (TRAIL)] to its corresponding death receptor (e.g. Fas and TRAIL receptors DR4 and DR5), which lead to a sequential activation of caspase- 8 and -3 . The intrinsic pathway is characterized by the collapse of $\Delta \Psi \mathrm{m}$, causing the release of cytochrome $c$ or Smac/DIABLO from the mitochondrial intermembrane space to the cytosol and the formation of the apoptosome complex consisting of cytochrome $c$, Apaf-1 and caspase-9. Then, caspase-9 is activated at the apoptosome and in turn activates caspase-3, committing the cell to apoptosis. It has been well documented that HNK-induced apoptosis is characterized by the activation of caspase- $3,-8$ and -9 in human multiple myeloma cells and B-cell chronic lymphocytic leukemia cells $(18,25)$. However, whether the same phenomenon as that of the above mentioned cell lines occurs in glioblastoma cells remains to be elucidated. In the present study, we noted that treatment of U87 with HNK did result in pronounced increase in caspase- 8 activation, as well as a significant elevation in Fas and FasL expression, which highlighted the involvement of the extrinsic pathway. Furthermore, we observed increased cleaved fragments of caspase- 9 and loss of $\Delta \Psi$ after HNK treatment in U87 cells, similar to previous reports in other cell types $(18,25)$. The Bcl-2 family of proteins plays an important role in the regulation of mitochondrialmediated apoptosis (26). The family consists of anti-apoptotic proteins, such as Bcl-2 and $\mathrm{Bcl}-\mathrm{x}_{\mathrm{L}}$, as well as pro-apoptotic members, such as Bax, Bid, Bax and Bak. Accumulating evidence suggests it is the relative ratio of anti-apoptotic and pro-apoptotic Bcl-2 family proteins rather than the levels of individual proteins that plays a major role in determining the survival or death of cells (27). Consistent with a previous report on human colorectal cells (6), our data indicated that HNK increased expression of Bax and decreased expression of Bcl-2, finally resulting in downregulation of Bcl-2/Bax ratio and further confirmed that the intrinsic apoptotic pathway is also involved in HNK-induced apoptosis in U87 cells.

A previous study revealed that both caspase-dependent and -independent mechanisms are involved in HNK-induced apoptosis in human multiple myeloma (18). Similarly, we found that pretreatment with the pan-caspase inhibitor Z-VAD-FMK completely abrogated the cleaved caspase- 3 fragments, indicating that caspase activation may be fully blocked by pan-caspase inhibitor. The apoptosis induced by HNK was not accordingly abrogated by addition of the pan-caspase inhibitor Z-VAD-FMK, which suggests that the caspase-independent pathway may also be involved in apoptosis induced by HNK in U87 cells except for caspase-dependent pathway.

Numerous studies have indicated that HNK can stimulate apoptosis by modulating multiple signaling pathways including nuclear factor- $\mathrm{\kappa B}(\mathrm{NF}-\kappa \mathrm{B})$, signal transducers and activator of transcription 3 (STAT3), mammalian target of rapamycin (m-TOR) which have considerable relevance during cancer initiation and progression (4). However, the role of MAPK and STAT3 signaling pathway in HNK-induced apoptosis in U87 glioblastoma cells has never been examined. We then focused on the status of STAT3 and MAPK activation in HNK-treated glioblastoma cells.

MAPK families play an important role in proliferation, differentiation, development, transformation and apoptosis (28). At least three MAPK families have been studied in detail: extracellular signal-regulated kinases (ERKs), c-Jun NH2-terminal kinases (JNKs) and p38 MAPKs (29). It has been reported that activated mitogen-activated protein kinase MAPK cascade leading to ERK $1 / 2$ phosphorylation is expressed in the majority of glial neoplasms and pharmacological targeting of the activated MEK/ERK1/2 module with the MEK inhibitor U0126 attenuates cell proliferation (30). Contrary to ERK1/2 MAPK, in some cases the stress-activated protein kinase (SAPK, also known as JNK; c-Jun amino-terminal kinase) was revealed to be activated in response to stress stimuli such as drug treatment (31). It has been reported that mimosine (32) and scriptaid (33) could induce apoptosis and inhibit glioma cell proliferation by elevating JNK activation. In the present study, we found the levels of phosphorylated ERK1/2 decreased in a time-dependent manner following HNK treatment, suggesting that HNK-induced apoptosis in U87 cells may be mediated by 
ERK1/2 downregulation. In contrast to previous studies, we detected no changes in phosphorylated JNK, which means SAPK/JNK may not contribute to apoptosis induced by HNK in U87 cells. Regarding another MAPK family-p38, the downstream effect of ROS accumulation, several studies revealed that activation of p38 may be required for apoptosis in human leukemic cells $(34,35)$. The present study showed the same phenomenon occurred in U87 cells after treatment with HNK. We speculate a quick burst of $\operatorname{ROS}(9,36)$ may account for the activation of p38 in other cells treated with HNK, although we did not determine the modulation of ROS level in this study.

It has been confirmed that high STAT3 activity is frequently present in human tumors, including glioblastoma(19). Activation of STAT3 and its target genes, such as c-myc (37), survivin (21), bcl-2, can lead to cell-cycle progression, pro-angiogenesis, anti- apoptotic effects, tumor invasion and metastasis (20). Thus, STAT3 has emerged as a promising molecular target for glioblastoma therapy. A previous study revealed that blockage of the STAT3 signaling pathway with a decoy oligonucleotide suppresses growth of human malignant glioma cells (38). HNK was reported to inhibit the STAT3 signaling pathway in hepatocellular carcinoma cells (39). Here, we observed significant downregulation of constitutive STAT3 activation is in line with apoptosis exhibited through modulation of cleaved caspase-3 protein. In addition, survivin, a downstream target of STAT3, accordingly decreased. Collectively, STAT3 signaling may be involved in apoptosis induced by HNK in U87 cells.

In conclusion, the present study confirmed that HNK inhibited proliferation of glioblastoma cells by causing a slight $\mathrm{G}_{0} / \mathrm{G}_{1}$ phase cell cycle arrest and inducing apoptosis. Here, we demonstrated for the first time that HNK triggered apoptosis of glioblastoma cells through both caspase-independent and -dependent pathways; the latter was initiated by the extrinsic and intrinsic pathway. Moreover, the inhibition of STAT3 signaling, ERK1/2 as well as activation of p38 MAPK signaling pathway may be involved in apoptosis induced by HNK in U87 cells. Whether STAT3 and MAPK signaling are necessary for apoptosis should be further examined.

\section{Acknowledgements}

The authors thank Professor Xiao Wang (Shandong Analysis and Test Center, Shandong Academy of Sciences) for providing purified Honokiol and Dr Lingzhi Huang for revising the manuscript. This study was supported by the Natural Science Foundation of China (81172792 and 81101605), the Natural Science Foundation of Shandong Province (ZR2011HL045, ZR2010HQ034 and ZR2011HL050), the Science and Technology Development Grant of the State Administration of Traditional Chinese Medicine of Shandong Province (2011-234) and the Doctor's Foundation of Shandong Province (BS2009YY008).

\section{References}

1. Dolecek TA, Propp JM, Stroup NE and Kruchko C: CBTRUS statistical report: primary brain and central nervous system tumors diagnosed in the United States in 2005-2009. Neuro Oncol 14: (Suppl 5): v1-v49, 2012.

2. Gauden AJ, Hunn A, Erasmus A, Waites P, Dubey A and Gauden SJ: Combined modality treatment of newly diagnosed glioblastoma multiforme in a regional neurosurgical centre. J Clin Neurosci 16: 1174-1179, 2009.
3. Jansen M, Yip S and Louis DN: Molecular pathology in adult gliomas: diagnostic, prognostic, and predictive markers. Lancet Neurol 9: 717-726, 2010.

4. Arora S, Singh S, Piazza GA, Contreras CM, Panyam J and Singh AP: Honokiol: a novel natural agent for cancer prevention and therapy. Curr Mol Med 12: 1244-1252, 2012.

5. Ishikawa C, Arbiser JL and Mori N: Honokiol induces cell cycle arrest and apoptosis via inhibition of survival signals in adult T-cell leukemia. Biochim Biophys Acta 1820: 879-887, 2012.

6. Wang T, Chen F, Chen Z, Wu YF, Xu XL, Zheng S and Hu X: Honokiol induces apoptosis through p53-independent pathway in human colorectal cell line RKO. World J Gastroenterol 10: 2205-2208, 2004.

7. Arora S, Bhardwaj A, Srivastava SK, Singh S, McClellan S, Wang B and Singh AP: Honokiol arrests cell cycle, induces apoptosis, and potentiates the cytotoxic effect of gemcitabine in human pancreatic cancer cells. PLoS One 6: e21573, 2011.

8. Wolf I, O'Kelly J, Wakimoto N, et al: Honokiol, a natural biphenyl, inhibits in vitro and in vivo growth of breast cancer through induction of apoptosis and cell cycle arrest. Int $\mathbf{J}$ Oncol 30: 1529-1537, 2007.

9. Hahm ER and Singh SV: Honokiol causes $G_{0}-G_{1}$ phase cell cycle arrest in human prostate cancer cells in association with suppression of retinoblastoma protein level/phosphorylation and inhibition of E2F1 transcriptional activity. Mol Cancer Ther 6: 2686-2695, 2007.

10. Wang Y, Yang Z and Zhao X: Honokiol induces paraptosis and apoptosis and exhibits schedule-dependent synergy in combination with imatinib in human leukemia cells. Toxicol Mech Methods 20: 234-241, 2010.

11. Kaushik G, Ramalingam S, Subramaniam D, et al: Honokiol induces cytotoxic and cytostatic effects in malignant melanoma cancer cells. Am J Surg 204: 868-873, 2012.

12. Wang X, Duan X, Yang G, et al: Honokiol crosses BBB and $\mathrm{BCSFB}$, and inhibits brain tumor growth in rat $9 \mathrm{~L}$ intracerebral gliosarcoma model and human U251 xenograft glioma model. PLoS One 6: e18490, 2011.

13. Jeong JJ, Lee JH, Chang KC and Kim HJ: Honokiol exerts an anticancer effect in T98G human glioblastoma cells through the induction of apoptosis and the regulation of adhesion molecules. Int J Oncol 41: 1358-1364, 2012.

14. Ren X, Zhang Y, Li C, et al: Enhancement of baicalin by hexamethylene bisacetamide on the induction of apoptosis contributes to simultaneous activation of the intrinsic and extrinsic apoptotic pathways in human leukemia cells. Oncol Rep 30: 2071-2080, 2013.

15. Liu J, Bi G, Wen P, et al: Down-regulation of CD44 contributes to the differentiation of HL-60 cells induced by ATRA or HMBA. Cell Mol Immunol 4: 59-63, 2007.

16. Riedl SJ and Shi Y: Molecular mechanisms of caspase regulation during apoptosis. Nat Rev Mol Cell Biol 5: 897-907, 2004.

17. Shimizu S, Narita M and Tsujimoto Y: Bcl-2 family proteins regulate the release of apoptogenic cytochrome $c$ by the mitochondrial channel VDAC. Nature 399: 483-487, 1999.

18. Ishitsuka K, Hideshima T, Hamasaki M, et al: Honokiol overcomes conventional drug resistance in human multiple myeloma by induction of caspase-dependent and -independent apoptosis. Blood 106: 1794-1800, 2005.

19. Rahaman SO, Harbor PC, Chernova O, Barnett GH, Vogelbaum MA and Haque SJ: Inhibition of constitutively active Stat 3 suppresses proliferation and induces apoptosis in glioblastoma multiforme cells. Oncogene 21: 8404-8413, 2002.

20. Yu H and Jove R: The STATs of cancer - new molecular targets come of age. Nat Rev Cancer 4: 97-105, 2004.

21. Zhou J, Bi C, Janakakumara JV, et al: Enhanced activation of STAT pathways and overexpression of survivin confer resistance to FLT3 inhibitors and could be therapeutic targets in AML. Blood 113: 4052-4062, 2009.

22. Ohtsubo M, Theodoras AM, Schumacher J, Roberts JM and Pagano M: Human cyclin E, a nuclear protein essential for the G1-to-S phase transition. Mol Cell Biol 15: 2612-2624, 1995.

23. Ullah Z, Lee CY and Depamphilis ML: Cip/Kip cyclin-dependent protein kinase inhibitors and the road to polyploidy. Cell Div 4: $10,2009$.

24. Constantinou C, Papas KA and Constantinou AI: Caspaseindependent pathways of programmed cell death: the unraveling of new targets of cancer therapy? Curr Cancer Drug Targets 9: 717-728, 2009.

25. Battle TE, Arbiser J and Frank DA: The natural product honokiol induces caspase-dependent apoptosis in B-cell chronic lymphocytic leukemia (B-CLL) cells. Blood 106: 690-697, 2005. 
26. Desagher S and Martinou JC: Mitochondria as the central control point of apoptosis. Trends Cell Biol 10: 369-377, 2000.

27. Reed JC, Jurgensmeier JM and Matsuyama S: Bcl-2 family proteins and mitochondria. Biochim Biophys Acta 1366: 127-137, 1998.

28. Ramos S: Cancer chemoprevention and chemotherapy: dietary polyphenols and signalling pathways. Mol Nutr Food Res 52: 507-526, 2008.

29. Zhang W and Liu HT: MAPK signal pathways in the regulation of cell proliferation in mammalian cells. Cell Res 12: 9-18, 2002

30. Glassmann A, Reichmann K, Scheffler B, Glas M, Veit N and Probstmeier R: Pharmacological targeting of the constitutively activated MEK/MAPK-dependent signaling pathway in glioma cells inhibits cell proliferation and migration. Int J Oncol 39: $1567-1575,2011$

31. Kyriakis JM and Avruch J: Mammalian mitogen-activated protein kinase signal transduction pathways activated by stress and inflammation. Physiol Rev 81: 807-869, 2001.

32. Qiao S, Murakami K, Zhao Q, et al: Mimosine-induced apoptosis in C6 glioma cells requires the release of mitochondria-derived reactive oxygen species and p38, JNK activation. Neurochem Res 37: 417-427, 2012.

33. Sharma V, Koul N, Joseph C, Dixit D, Ghosh S and Sen E: HDAC inhibitor, scriptaid, induces glioma cell apoptosis through JNK activation and inhibits telomerase activity. J Cell Mol Med 14: $2151-2161,2010$
34. Huh JE, Kang KS, Chae C, Kim HM, Ahn KS and Kim SH: Roles of $\mathrm{p} 38$ and JNK mitogen-activated protein kinase pathways during cantharidin-induced apoptosis in U937 cells. Biochem Pharmacol 67: 1811-1818, 2004.

35. Konopleva M, Contractor R, Kurinna SM, Chen W, Andreeff M and Ruvolo PP: The novel triterpenoid CDDO-Me suppresses MAPK pathways and promotes p38 activation in acute myeloid leukemia cells. Leukemia 19: 1350-1354, 2005

36. Han LL, Xie LP, Li LH, Zhang XW, Zhang RQ and Wang HZ: Reactive oxygen species production and $\mathrm{Bax} / \mathrm{Bcl}-2$ regulation in honokiol-induced apoptosis in human hepatocellular carcinoma SMMC-7721 cells. Environ Toxicol Pharmacol 28: 97-103, 2009.

37. Stearns D, Chaudhry A, Abel TW, Burger PC, Dang CV and Eberhart CG: c-myc overexpression causes anaplasia in medulloblastoma. Cancer Res 66: 673-681, 2006.

38. Gu J, Li G, Sun T, et al: Blockage of the STAT3 signaling pathway with a decoy oligonucleotide suppresses growth of human malignant glioma cells. J Neurooncol 89: 9-17, 2008.

39. Rajendran P, Li F, Shanmugam MK, et al: Honokiol inhibits signal transducer and activator of transcription-3 signaling, proliferation, and survival of hepatocellular carcinoma cells via the protein tyrosine phosphatase SHP-1. J Cell Physiol 227: 2184-2195, 2012. 